\title{
ILALR: \\ AN INCREMENTAL GENERATOR OF LALR(1) PARSERS
}

\author{
R. Nigel Horspool \\ Department of Computer Science \\ University of Victoria, P.O. Box 1700 \\ Victoria, B.C., Canada V8W 2 Y2
}

\begin{abstract}
An incremental parser generator would be a desirable tool for use in compiler implementation projects or for experimental use in designing new languages. It would allow the tool user to incrementally add or remove production rules from a grammar while maintaining correct parse tables, and would report grammatical problems to the user at the earliest possible moment. ILALR is such an incremental parser generator for the LALR(1) class of grammars. It uses incremental algorithms for computing the LALR(1) parser tables.
\end{abstract}

Keywords and Key Phrases: parsers, LALR(1), compilers, incremental algorithms.

\section{Introduction}

In the bottom-up approach to compiler implementation, the compiler writer must create a grammar for the language and attach semantic actions to its production rules. The grammar is prepared in a computer file, typically using a notation based on BNF, and then the compiler writer uses a parser generator to create a parser from the grammar file. Different parser generators accept different classes of grammars. Examples of parser generators include yacc [9], llama [5] and lalrgen [3] which accept the LALR(1) class of grammars, and llgen [3] for the LL(1) class of grammars.

The compiler writer can rarely use a published grammar for the language such a grammar is usually provided in a form more suitable for humans to read. The published grammar may suffer from ambiguities (resolved by separate written explanations), or it may not belong to a grammar class accepted by the available parser generators. Therefore, the compiler writer usually has to go through a process of grammar debugging. This is an iterative process of editing the grammar file, executing the parser generator to obtain error messages, re-editing and massaging the grammar rules to remove the problems and re-trying. A programming language designer might also like to ensure that he/she has an unambiguous grammar and this person would use a parser generator to check out and debug potential BNF grammar definitions. Grammar debugging can be a tedious and frustrating experience, especially for novices.

It would appear that the ideal solution to the grammar debugging problem would be an incremental parser generator. Such a software tool would operate interactively, permitting the user to modify a grammar one rule at a time and 
reporting problems to the user as soon as they become apparent. This tool would be more convenient because there is no need to alternate between separate editor and parser generator programs. It would also make it easier for the user to understand problems with the grammar, because the error would be reported immediately the user adds a rule that, for example, causes the grammar to become ambiguous.

There appears to have been little previous work on incremental generation of parsers. For the LR parsing methods, a doctoral dissertation [4] and a forthcoming technical report [6] exist. They are mentioned at the end of this paper. Also, some older work $[7,12]$ has been published on incremental parser generation for the much smaller LL(1) class of grammars.

\section{Basic Design Issues}

An important issue to be decided is the unit of change for the grammar. At one extreme, we can imagine a tool that re-checks the grammar each time the user changes a single character in the grammar file. At the other extreme, the tool might re-check only after a complete set of production rules with a common left-hand side have been added or removed. We decided that the most convenient unit of change would be one production rule.

But if we re-check after each rule addition or deletion, our tool has to be. prepared to accept (temporarily) an incomplete grammar. The symptoms of an incomplete grammar are that some rules may be unreachable and some non-terminal symbols may be useless. Algorithms for detecting (and removing) unreachable rules and useless non-terminals exist [1] and are often included in parser generators. In the case of ilalr, these algorithms are only applied at the explicit request of the user or when the user asks for the parser to be generated.

To make ilalr as flexible as is reasonably possible, the user is allowed to add or delete production rules in absolutely any order. Furthermore, the start symbol of the grammar can be specified at any time - or even be changed. Nor does ilalr require the user to specify which symbols are terminals and which are nonterminals. Why should it, when such information can be inferred from the complete grammar? (But ilalr will accept this information and use it for error-checking purposes if the user wishes to provide it.)

\section{The General Approach}

Suppose that the user has added the following rules to the grammar and has not yet specified a start symbol.

$\begin{array}{lll}\text { S } & ::= & \text { begin SL end } \\ \text { SL } & ::= & \text { SL } ; \text { STMT } \\ \text { ASMT } & ::= & \text { VAR }:=\text { EXPR } \\ \text { VAR } & ::= & \text { ident }\end{array}$

In this example, S, SL, ASMT and VAR must be non-terminal symbols. But it is unclear whether STMT or even begin represents a terminal or non-terminal symbol. Therefore, ilalr assumes that any symbol which does not currently occur on the left-hand side of some rule must be a terminal symbol. To make any sense of the example grammar, we must consider it to define a language of sentential forms (as opposed to a language of sentences containing only terminal symbols). And in the absence of an explicit declaration, we must consider every non-terminal symbol as a 
potential goal symbol for the grammar. Thus, the example grammar describes a language that includes the sentential forms:

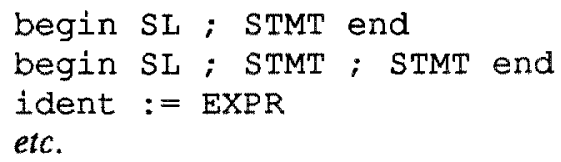

In order to handle the multiple potential goal symbols, ilalr introduces a fictitious goal symbol $\mathrm{S}^{*}$, which we call the supergoal. For each symbol $\mathrm{N}$ that is known to be a non-terminal, ilalr adds an implicit production rule

$$
\mathrm{S}^{*} \rightarrow \$_{\mathrm{N}} \mathrm{N} \$_{\mathrm{N}}
$$

where $\$_{N}$ represents a unique delimiter symbol. Normal grammar analysis techniques can be applied to the augmented grammar using $S^{*}$ as the goal symbol. Unique delimiters are required around all the non-terminals because of potential conflicts during LR analysis.

While a grammar is incomplete, ilalr cannot know for certain whether a symbol is nullable or not. All the user has to add is one a rule like

$$
\mathrm{X} \quad::=\langle\operatorname{empty}\rangle
$$

which causes $\mathrm{X}$ and, perhaps, several other symbols to suddenly become nullable. Ilalr assumes that all symbols are non-nullable unless nullability can be deduced from the grammar rules. The opposite approach of assuming all symbols to be nullable would cause many spurious error messages to be reported while a grammar is being developed.

\section{Incremental LALR(1) Algorithms}

Ilalr implements incremental forms of LALR(1) parser generator algorithms. At all times, it maintains the LR(0) sets of items for the grammar and a LALR(1) lookahead set for each item. To help compute the lookahead sets, ilalr also maintains a set $N U L L$ that contains all the symbols known to be nullable and it maintains starters sets for the symbols. The starters set for symbol $X, S T A R T(X)$, is defined as $\{Y \mid X \Rightarrow Y \alpha\}$

\subsection{Addition of a New Rule}

When a new rule, $A::=\times 1 \times 2 \ldots \times n(n \geq 0)$, is added to a grammar $G$, a new grammar $G^{\prime}$ is created. If we consider a grammar $G$ to be defined by the quadruple $\langle\mathrm{T}, \mathrm{N}, \mathrm{P}, \mathrm{S}\rangle$, then $\mathrm{G}^{\prime}$ is a modified quadruple $\left\langle\mathrm{T}^{\prime}, \mathrm{N}^{\prime}, \mathrm{P}^{\prime}, \mathrm{S}^{\prime}\right\rangle$, where

$$
\begin{array}{ll}
N^{\prime} & =N \cup\{A\} \\
T^{\prime} & =T \cup\left\{\$_{A^{\prime}} X 1, X 2, \ldots, X n\right\}-N^{\prime} \\
S^{\prime} & =S^{*} \\
P^{\prime} & =P \cup\left\{A \rightarrow X 1 X 2 \ldots X n, S^{*} \rightarrow \$_{A} A S_{A}\right\}
\end{array}
$$

Note that $G$ and $G^{\prime}$ represent the grammars actually being analyzed by ilalr rather than the grammar as entered by the user. That is, $G$ and $G^{\prime}$ include the extra symbols and implicit goal production rules that are added by ilalr. If ilalr starts with an initially empty grammar, $\left\langle\phi, \phi, \mathrm{S}^{*}, \phi>\right.$, then the grammar held by ilalr after a sequence of rule additions is completely determined. 
Algorithms for computing NULL, START and LALR lookahead sets are simplified by the observation that these sets grow monotonically when new rules are added to the grammar. However (unless some unnatural definitions are used), we cannot consider the graph of states for the LR( 0$)$ recognizer as growing monotonically.

In particular, ilalr can use a simple iterative algorithm for recomputing NULL, the set of nullable symbols, after a rule addition. (The algorithm is so simple that it is omitted.)

If it turns out that NULL' $=$ NULL, there is a simple algorithm for updating the starters sets. It is expressed by the following equations:

$$
\operatorname{START}^{\prime}(C)= \begin{cases}\operatorname{START}(C) \cup \operatorname{START}(X 1 X 2 \cdots X n) & \text { if } A \in \operatorname{START}(C) \\ \operatorname{START}(C) & \text { otherwise }\end{cases}
$$

where we use a definition of START extended by:

$\operatorname{START}(Y 1 Y 2 \cdots Y k)= \begin{cases}\operatorname{START}(Y 1) & \text { if } Y 1 \notin N U L L^{\prime} \text { and } k \geq 2 \\ \operatorname{START}(Y 1) \cup \operatorname{START}(Y 2 \cdots Y k) & \text { if } Y 1 \in N U L L^{\prime} \text { and } k \geq 2\end{cases}$

We do not have such an efficient algorithm to handle the situation when NULL' $\neq$ NULL. In this case, ilalr uses a simple iterative algorithm, taking advantage of the monotonic nature of the problem.

Updating the LR(0) sets of items for the grammar is less trivial. The LR(0) recognizers for $G^{\prime}$ and $G$ are closely related; in that for every state in $G$ we can find one or more corresponding states in $\mathrm{G}^{\prime}$. But there does not appear to be an easy algorithm to tell when a state in $\mathrm{G}$ must map to two or more states in $\mathrm{G}^{\prime}$. Here is an example. Suppose that $\mathrm{G}$ is the grammar

$\begin{array}{llllll}S & ::= & \text { C } & \text { D } & \text { I } & \text { E } \\ \text { C } & ::=\text { a A b } & & \\ \text { D } & ::=\text { a e c } & & \\ \text { B } & ::=\text { e d } & & \\ \text { E } & ::=\text { f D } & & \end{array}$

Now, amongst about 20 other states, the $L R(0)$ recognizer for $G$ will include the states and transitions shown below.

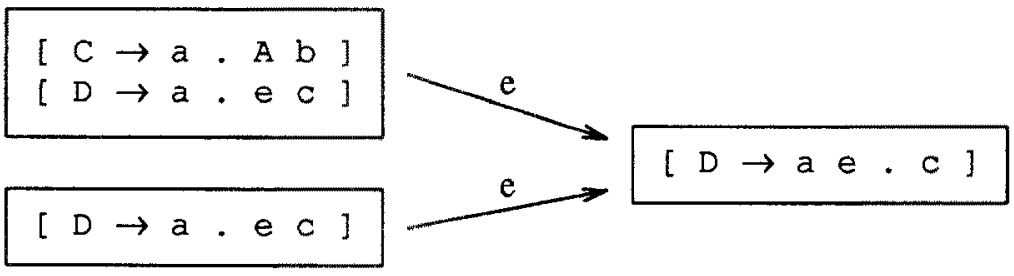

If we now add the rule, 'A : : = B', the corresponding states in the new LR(0) recognizer are 


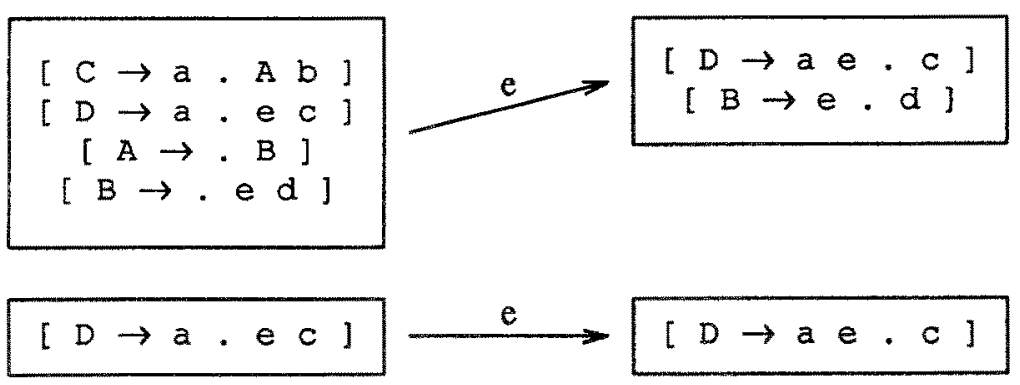

That is, the state on the right has split to become two states. But if the 'B : : = e d' rule had not been in the grammar, the state would not have split. This demonstrates that we cannot determine how to update the set of items for a state or whether to split that state unless we have already updated all the predecessor states.

The incremental algorithm implemented in ilalr begins by using an approximate method for modifying sets of items. It is approximate because it does not split states, implying that not all the states needed for the new recognizer are created, and it may create some sets of items that do not correspond to any states in the new recognizer. Missing states must be added by invoking a state closure algorithm. Extraneous sets of items are removed by a marking algorithm that checks to see which states are reachable from the start state. (Unless memory is in short supply, there is no need to invoke the marking algorithm after every rule addition.) As long as the mapping process performs a reasonably accurate job, the overall efficiency is not badly compromised by the creation of unreachable states. In essence, the mapping algorithm simply checks the item sets associated with each state and (incrementally) re-performs the item closure operation. Thus, if the set of items for some state contains an item of the form $[\mathrm{x} \rightarrow \alpha$. $\mathrm{A} \beta]$, we add the new closure item $[\mathrm{A} \rightarrow . \mathrm{X} 1 \mathrm{X} 2 \ldots \mathrm{Xn}]$ (and more closure items if $\mathrm{X} 1$ is a nonterminal symbol). Addition of closure items is implemented by an iterative algorithm based on a worklist implementation.

Updating the LALR(1) lookahead sets is a difficult problem because these sets do not necessarily grow monotonically. After a rule addition, some sets may contract in size. Informally, this occurs when a state in the old recognizer is split and then the old lookahead set is shared between the two new recognizer states. Our solution is to operate under the assumption that the lookahead sets cannot contract in size (but be prepared to correct the situation). Ilalr uses a simple worklist-oriented algorithm. In this algorithm, a change to the lookahead set for one item in a state ST causes that change to be propagated to closure items in the same state and causes affected states reached by transitions from ST to be added to the worklist (and thus they will later be updated themselves).

If a LALR(1) conflict is discovered in some state, this conflict may have been caused by our incorrect assumption, above. In this case, ilalr clears all the lookahead sets to empty and re-computes them correctly (using the worklist algorithm). The user is wamed of a problem with the grammar only if the conflict still exists after the re-computation. It should be noted that this re-computation of lookahead sets is never required for a SLR(1) grammar. 


\subsection{Removing a Production Rule}

Deletion of a rule from the grammar is a little harder to implement than addition, and the algorithms are also less efficient. But, since rule deletion is likely to be a less frequent occurrence than rule addition, the asymmetry in efficiency is not relevant. Here, in outline, are the methods used in ilalr to handle deletion of a rule, A $\rightarrow \mathrm{x} 1 \mathrm{x} 2 \ldots \mathrm{xn}$, from the grammar. First, no update to the NULL set is required if $A$ is currently non-nullable or if one of $x 1, x 2, \ldots X n$ is non-nullable. Otherwise, ilalr recomputes NULL from scratch.

Modification of the START sets can be performed reasonably efficiently if no symbols change their nullability because of the deletion. The updates are performed in two stages. First, if START(X) contains $A$, for any $X$, then the members of START(A) are all removed from START(X). However, this will usually remove too many elements and then an iterative algorithm (the same one mentioned for the action of adding a rule) is used to re-check the sets and add missing elements back.

Updating the $L R(0)$ recognizer requires removal of some states and slimming many item sets down. Using an approach similar to that used for adding a rule to the grammar, we perform an approximate mapping process. The process considers kernel and completion items separately. Any kemel items formed from the deleted rule, with the marker in any position, are removed. All completion items formed from the deleted rule are deleted and any closure items for deleted items are themselves removed. As before, we must perform a state closure operation to add missing states and a reachability analysis is required to remove unreachable states.

The LALR(1) lookahead sets are computed only for items in new states that are added by the state closure step, above. This is likely to result in many lookahead sets containing extra elements (but not sets will be missing any elements). If the extra elements subsequently cause a conflict to be detected, the lookahead sets will be re-computed before any error is reported to the user.

\section{The User Interface}

The ilalr program includes interactive commands for these operations - rule addition; rule deletion; setting/changing the goal symbol; defining symbols to be terminals; reading commands from an external file; writing the current grammar and definitions to an external file; creating an external LALR(1) parse table file; checking the grammar for completeness; parsing a sample sentence; listing the grammar, its symbols, all rules with a particular left-hand side, the NULL set, the START sets, or the states of the LALR(1) recognizer.

As far as possible, the user commands adopt the notation used by the yacc parser generator on UNIX systems. Some features of yacc, such as declaratrion of operator precedences, are not supported by ilalr. But if the user avoids using the unsupported features, the same grammar file can be processed by either yacc or ilalr.

If the user adds a rule which causes a LALR(1) conflict, a brief message is generated which reports this fact. Although the conflict cannot go away if the user continues to add new rules, the warning message will not be repeated. However, as long as a conflict exists in the grammar, a different kind of input prompt is used to remind the user of the problem. The user can enter a special command at any time which asks ilalr to display example sentences that illustrate conflicts in the grammar. 
The output from any command that generates more than a few lines of output is piped through the UNIX more pager program. Thus the user can, for example, easily scan forward to a particular state in a listing of the parser states. (If the less pager is used, backward scanning is also possible.)

If a grammar is complete and free of conflicts, the user can create an external file that contains a machine-readable representation of the LR recognizer. A separate program, mkparser, is provided for translating that file into an executable LALR(1) parser. This parser interfaces with scanners created by lex[10] or mkscan [8].

\section{Experience}

At the time of writing, ilalr has been in use for about one year and has already been used successfully by two classes of undergraduate students taking a compiler construction course. All the students found the incremental approach to grammar analysis easy to understand. Those students who had had experience with other parser generators (typically yacc) remarked on how much easier ilalr was to use.

In terms of machine resource consumption, ilalr is relatively inefficient compared to competing tools such as yacc. For the analysis of a complete grammar, ilalr consumes considerably more CPU time than yacc. And because sizes of internal tables cannot be determined by a pre-pass through the grammar, less memoryefficient data structures must be used by ilalr. Another point that becomes apparent to the user when rules are read in from a file is that the amount of CPU time needed to process a new rule grows with the size of the grammar. Empirically, the maximum time required to process one new rule is approximately proportional to the square of the grammar size, but most rules are processed quickly. A new rule that causes several symbols to become nullable is particularly slow to process. But, even with a moderately large grammar (say 200 productions), ilalr executing on a SUN $3 / 280$ can process an additional rule faster than the user can type it. As an experimental observation, a grammar for the $\mathrm{C}$ language containing 219 production rules requires almost exactly three times as much CPU time with ilalr as with yacc.

The approximate procedure used to re-build the $L R(0)$ recognizer after a rule addition turns out to be surprisingly effective. For example, when ilalr read 83 rules from a test grammar (a grammar based on Wirth's PL/0 language), a recognizer with 241 states was eventually constructed. But in the course of building the 83 different recognizers, a total of only 40 unreachable states were created. The unreachable states only had to be removed when the final version of the parser tables were output.

\section{Comparison with Other Work}

Heering, Klint and Rekers [6] have a Lisp implementation of an incremental parser generator. It is intended for use by language designers. Their implementation uses a 'lazy evaluation' approach which constructs the LR $(0)$ states only as they are needed during a parse. Lookahead sets to resolve parser conflicts are not computed. Instead, a parallel parsing algorithm which attempts to follow all possible parses is used. For interactive testing of languages and grammars, this is an excellent approach. But it is unsuitable for use as a compiler construction tool because the complete set of parser states is not necessarily available and because the parallel parsing algorithm is too inefficient for use in a production compiler. 
The $\mathrm{PhD}$ dissertation of Fischer [4] describes an incremental parser generator, implemented in PL/I, that is similar in nature to ilalr. In some respects, his parser generator is more powerful because it handles the full LR(1) class of grammars. It, too, uses an approximate technique for computing the lookahead sets, using the infamous NQLALR method [2] for this purpose. If a conflict is detected, the correct lookahead sets are re-computed using Pager's lane tracing method [11]. If the conflict still exists afterwards but can be eliminated by splitting states, the states are split (thus the full LR(1) class of grammars can be handled). Without access to his implementation, it is hard to make any comparisons between Fischer's methods and those used in ilalr. It certainly appears that Fischer did not provide an interactive interface for his implementation.

\section{Conclusions}

As computers become faster, it seems to be an inescapable (and desirable) trend for software to become interactive and incremental in nature. We have already seen text editing software progress from batch mode editors to interactive line editors to interactive screen-oriented editors like vi or emacs. WYSIWYG word processing is another example of this trend. Ilalr represents the first incremental and interactive software tool applied to grammar analysis and parser generation. The design of ilalr has raised some interesting issues and its implementation has required the invention of new algorithms for LALR(1) grammar analysis.

Further work is needed to improve the user interface - perhaps to integrate it with an intelligent editor like emacs so that more sophisticated editing operations than adding or deleting one production to/from the grammar can be supported (without the user having to learn a new editing language). Further investigation is needed into finding efficient incremental algorithms; some of the algorithms used by ilalr could be improved. Also, it might be worthwhile to investigate whether it would be easier and, if so, how much easier it would be to handle a smaller class of grammars, such as the SLR(1) or LL(1) classes.

\section{References}

[1] Aho, A.V., and J.D. Ullman. The Theory of Parsing, Translation and Compiling. Prentice-Hall, 1972.

[2] DeRemer, F.L., and T.J. Pennello. Efficient Computation of LALR(1) LookAhead Sets. ACM Trans. on Prog. Lang. and Systems, 4, 4 (Oct. 1982), pp. 615-649.

[3] Fischer, C.N., and R.J. LeBlanc Jr. Crafting a Compiler, Benjamin/Cummings, Menlo Park, 1988.

[4] Fischer, G. Incremental LR(1) Parser Construction as an Aid to Syntactical Extensibility. PhD Dissertation, Tech. Report 102, University of Dortmund, 1980.

[5] Hayes, I., and K. Robinson. A Tutorial on Llama: A Pascal Translator Generator. Dept. of Computer Science, Univ. of New South Wales, June 1985.

[6] Heering, J., P. Klint and J. Rekers. Incremental generation of Parsers. Technical Report (to appear), Centre for Mathematics and Computer Science, 
Amsterdam, 1988.

[7] Heindel, L.E., and J.T. Roberto. LANGPAK - An Interactive Language Design System. American Elsevier, New York, 1975.

[8] Horspool, R.N., and M.R. Levy. Mkscan - An Interactive Scanner Generator. Software - Pract. and Exp. 17,6 (June 1987), pp. 369-378.

[9] Johnson, S.C. Yacc - Yet Another Compiler-Compiler. Computer Science Tech. Report 32, Bell Laboratories, 1975.

[10] Lesk, M.E., and E. Schmidt. Lex - A Lexical Analyzer Generator. Computer Science Tech. Report 39, Bell Laboratories, 1975.

[11] Pager, D. The Lane Tracing Algorithm and Ways of Enhancing Its Efficiency. Inf. Science 12, 1 (1977), pp. 19-42.

[12] Vidart, J. Extensions Syntaxiques dans une Contexte. PhD Dissertation, Département d'informatique, Université of Grenoble, 1974. 